\title{
Revue Des Doses D'exposition Et De La Justification Des Radiographies Standard En Pratique Pédiatrique Au Togo
}

\author{
M. Tchaou \\ G.N. Gnakadja
}

Service de Radiologie et Imagerie Médicale CHU de Lomé, Togo

B. N'timon

Service de Radiologie et Imagerie Médicale CHU KARA, Kara, Togo

L. Sonhaye

A. Amadou

M. Dansou

L.K. Agoda-Koussema

K. Adjenou

K. N'dakena

Service de Radiologie et Imagerie Médicale CHU de Lomé, Togo

doi: 10.19044/esj.2016.v12n24p223 URL:http://dx.doi.org/10.19044/esj.2016.v12n24p223

\begin{abstract}
Aims: To assess the justification of indications and exposure doses to children during radiographics.

Methods: Prospective study of 102 X-ray exams of children collected over a period of 6 months in the Department of Radiology of Kara Teaching Hospital.

Objectives: To assess the rationale guidance and exposure doses to children when standard radiographic examinations.

Methodology: prospective observational study of 102 standard radiographs (Rx) collected in the radiology department of the University Hospital of Kara on a 6 month period.

Results: Male children were predominant with a sex ratio boy / girl of 1.3. The predominant age group was the 5 to 10 years. Chest X-rays were the most practiced exam, with 43\%. According to the Guide of well practices of French Society of Radiology (SFR) and the French Society of Biophysics and Nuclear Medicine (SFBMN), $80 \%$ of exams were justified. The comparison of our results to diagnostic reference levels (DRLs) shows that $43 \%$ and $39 \%$ of standard X-rays had dosimetric values exceeds the RDLs respectively the entrance Dose (De) and Dose Surface Product (DSP).
\end{abstract}


Conclusion:Our study reveals that compliance guidance assessments to the proper use of medical imaging examinations guide was not always effective but satisfactory. The study dosimetric constants showed that the dose to children exceeded in a large proportion of the French and Belgian DRLs.

Keywords: Radiographic, Dose, justification, children, Togo

\section{Resume}

Objectifs : Evaluer la justification des indications et les doses d'exposition délivrées aux enfants lors des examens radiographiques standards.

Méthodologie : Etude prospective observationnelle de 102 radiographies standards (Rx) colligées dans le service de radiologie du CHU de Kara sur une période de 6 mois.

Résultats : La sex-ratio garçons- filles était de 1,3. La tranche d'âge de 5 à 10 ans était la plus représentée. Les radiographies du thorax étaient majoritaires ((43\%). 80\% des examens étaient justifiées selon le Guide de Bon Usage (GBU) établi par la Société Française de Radiologie (SFR) et la Société Française de Biophysique et de Médecine Nucléaire (SFBMN). En comparant nos résultats aux niveaux de référence diagnostique (NRD) il ressort que $43 \%$ et $39 \%$ des Rx standard avaient une valeur dosimétrique supérieure à la NRD respectivement pour la dose à l'entrée (De) et le produit dose surface (PDS).

Conclusion: Notre étude révèle que la conformité des indications des examens avec le guide de bon usage des examens d'imagerie médicale n'était pas toujours effective mais satisfaisante. L'étude des constantes dosimétriques a montré que la dose délivrée aux enfants excédait dans une grande proportion les NRD français et belges.

Mots clé : Radiographie, Dose, justification, enfants, Togo

\section{Introduction}

La radioprotection est l'ensemble des principes et des moyens pouvant être mis en œuvre pour protéger les personnes exposées aux radiations ionisantes (Service de la prévention et de la sécurité, université du Québec, 2010). En effet, la protection des personnes exposées à des rayonnements ionisants à des fins médicales et médicolégales est, en France, réglementée par le décret $\mathrm{n}^{\circ}$ 2003-270 du 24 mars 2003, pris en application de la directive 97-43 Euratom du Conseil du 30 juin 1997 relative à la protection sanitaire des personnes contre les dangers des rayonnements ionisants lors d'expositions à des fins médicales (Vrousos C et al., 2004). La connaissance de doses délivrées lors des examens de radiologie diagnostique 
est une obligation légale et un impératif absolu de bonne pratique, préalable obligatoire de la démarche de justification et d'optimisation.

Les pratiques de radioprotection des patients sont par contre peu documentées en Afrique subsaharienne et notamment au Togo, en raison de l'absence ou de la mise en œuvre approximative des lois et règlements qui y sont relatifs (Ongolo-Zogo P et al., 2012).

$\mathrm{Au}$ Togo, il n’existe pas encore de texte juridique ou règlementaire dans la surveillance des niveaux d'exposition aux rayonnements ionisants (RI). Ceci explique l'absence de référence nationale fixant les niveaux de dose à ne pas dépasser.

La nécessité de réduire au minimum les doses reçues lors d'examens s’impose en radiologie diagnostique et plus encore en radiopédiatrie, puisque les enfants sont particulièrement sensibles aux risques liés aux rayonnements ionisants parce que d'une part leur corps est plus petit et que d'autre part leurs organes sont en croissance. En outre, au-delà des bonnes pratiques médicales et de la justification de l'acte, optimiser les examens suppose de mieux connaître les doses délivrées et les risques réellement encourus.

Ce travail investiguait de fait, la justification et l'optimisation (principes de la radioprotection des patients) en radiographie standard et scanographie pédiatrique au Centre Hospitalier Universitaire de Kara. Son objectif général était l'évaluation du niveau de respect des principes et normes de radioprotection en radiologie diagnostique chez les enfants ; les objectifs spécifiques étant d'évaluer la justification des indications des examens de radiographie standard et l’optimisation des procédures.

\section{Methodologie}

Il s'est agi d'une étude monocentrique, prospective descriptive, observationnelle qui s’était déroulée de décembre 2014 à juin 2015 dans le service de radiologie et imagerie médicale du CHU de Kara. Elle avait concerné tous les patients âgés de 0 à 15 ans ayant passé un examen de radiographie standard durant la période d’étude. Les examens étaient réalisés sur deux appareils: une table os - poumons, de marque STEPHANIX, fonctionnelle depuis Décembre 2003 et une table télécommandée de marque GENERAL ELECTRIC, fonctionnelle depuis Juillet 2010.

Les paramètres étudiés portaient sur le patient (âge, sexe, région anatomique explorée), la demande de l'examen (demandeur, indication, justification de l'examen sur la base du guide de bon usage de la Société Française de Radiologie) ; les paramètres liés à la procédure (, la Distance Foyer Film ou DFF, la Distance Foyer Peau ou DFP, l'utilisation de dispositifs de protection, la reprise ou non de l'examen), les constantes dosimétriques (Dose à l'entrée ou De, le produit dose surface ou PDS). 


\section{Resultats}

Un total de 1682 examens de radiographie standard ont été réalisés au CHU Kara pendant notre période d'étude dont 102 ont été inclus dans notre étude soit un pourcentage de $6.06 \%$.

Notre population d'étude était composée majoritairement de garçons (59 soit $58 \%$ ), et de 43 filles soit $42 \%$. La sex ratio garçons- filles était de 1,3 .

La figure 1 présente le diagramme des âges des patients de notre étude, ayant bénéficié d'une radiographie standard, avec un pic dans la tranche d’âge [5 - 10[, les extrêmes étant de 7 jours et 15 ans.

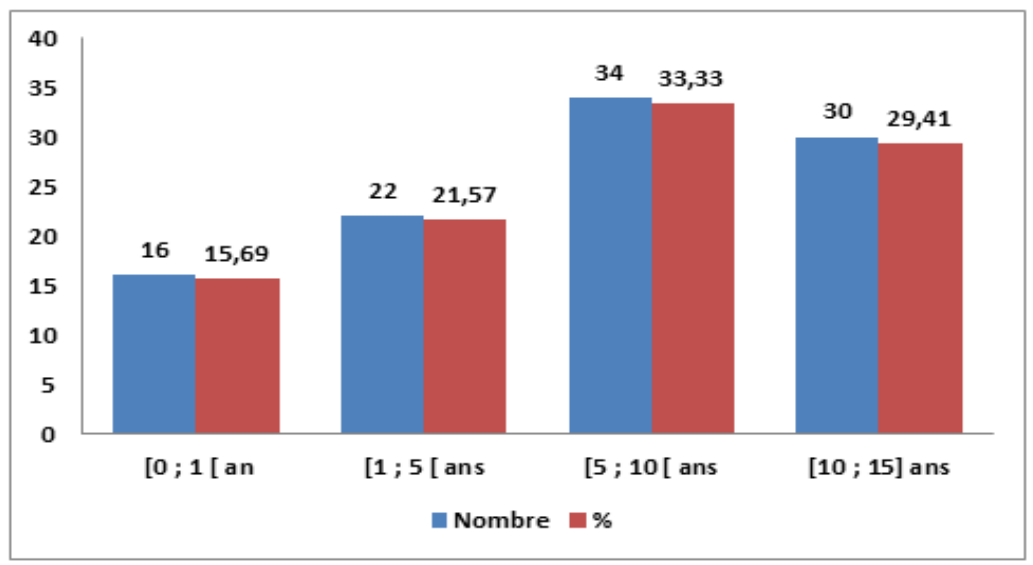

Figure 1: Diagramme des âges des enfants ayant bénéficié d’une Rx standard

Nous illustrons par la figure 2 la répartition des différentes régions explorées à la radiographie standard. Elle montre que la radiographie standard du thorax était l'examen le plus réalisé (43,14\%), suivi des radiographies des segments de membre.

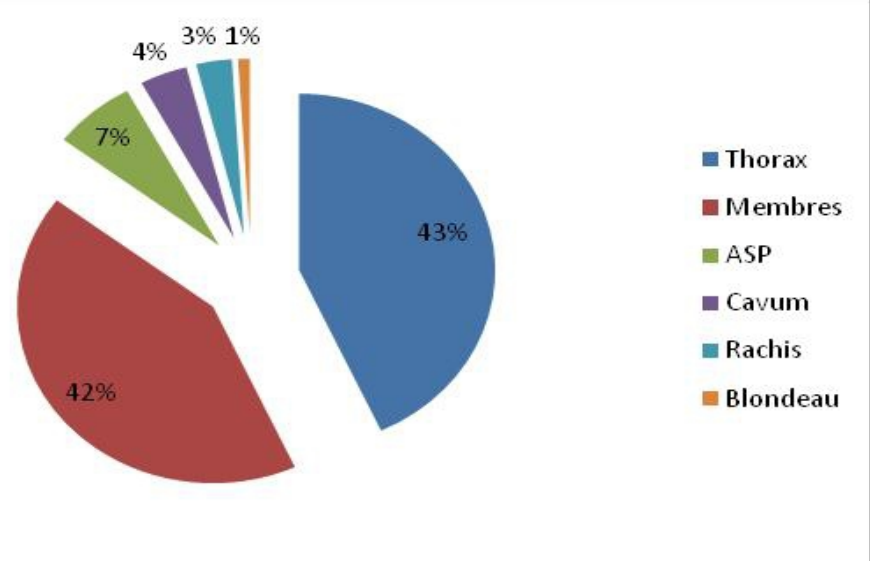

Figure 2 : Répartition des examens en fonction des régions et segments du corps explorés. 
Les indications les plus retrouvées étaient respectivement le traumatisme, la pneumopathie, le syndrome infectieux et la toux (Tableau I).

Tableau I: Répartition des examens en fonction de leurs indications

\begin{tabular}{ccc}
\hline & Nombre & Pourcentage \\
\hline Traumatisme & 39 & 38,24 \\
Pneumopathie & 13 & 12,75 \\
Syndrome infectieux & 12 & 11,76 \\
Toux & 11 & 10.79 \\
Dyspnée & 09 & 8,82 \\
Douleur thoracique & 04 & 3,92 \\
Malformation & 03 & 2,94 \\
Bilan & 03 & 2,94 \\
Autres & 03 & 2,94 \\
Ballonnement abdominal & 02 & 1,96 \\
Pathologie des VA* & 02 & 1,96 \\
Cardiopathie & 01 & 0.98 \\
Rachialgie & 01 & 0.98 \\
Station debout impossible & 01 & 0,98 \\
Sinusite & 01 & 0.98 \\
Total & 102 & 100 \\
\hline
\end{tabular}

* VA = végétations adénoïdes

Les assistants médicaux étaient les plus nombreux prescripteurs (71,57\%) suivis des médecins généralistes (13,73\%). (Tableau II)

Tableau II: Répartition des examens en fonction du grade des demandeurs

\begin{tabular}{ccc}
\hline & Nombre & Pourcentage \\
\hline Assistant Médical & 73 & 71,57 \\
Médecin Généraliste & 14 & 13,73 \\
Infirmier & 06 & 5,88 \\
Kinésithérapeute & 06 & 5,88 \\
Pédiatre & 02 & 1,96 \\
Cardiologue & 01 & 0,98 \\
Total & 102 & 100 \\
\hline
\end{tabular}

L'analyse de la justification des explorations radiographiques a été faite sur la base du guide de bon usage des examens d'imagerie médicale et a montré que quatre-vingt (80) \% des examens de radiographie standard étaient bien justifiés selon le GBU des examens d’imagerie médicale.

La majorité des examens demandés par les assistants médicaux était justifiée (figure 3). 


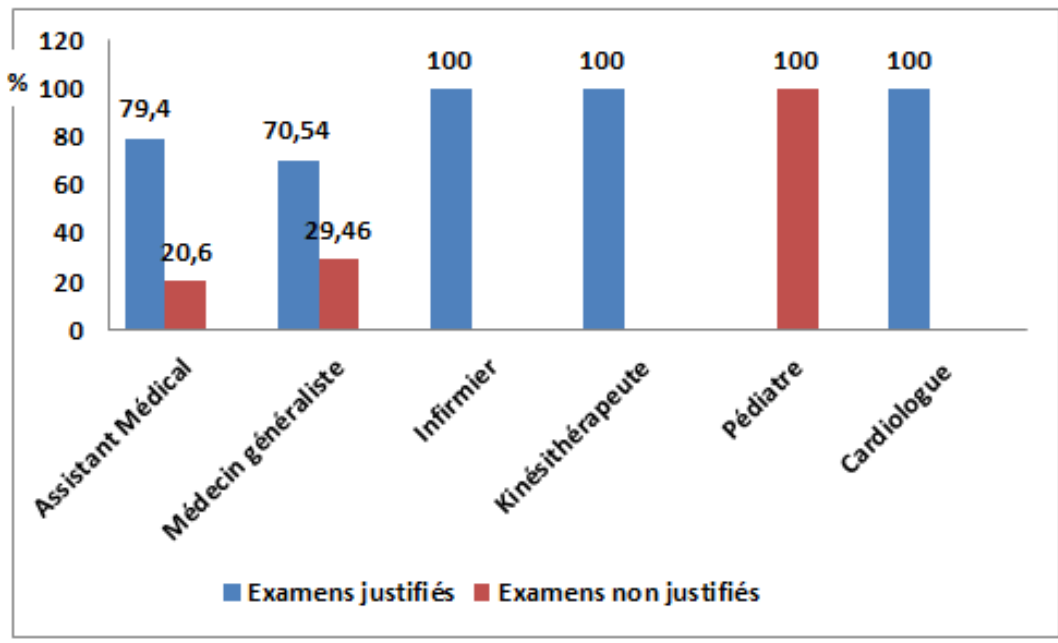

Figure 3 : Répartition des examens selon la qualification des demandeurs et le caractère justifié ou non de l'examen

A l'analyse des procédures, il ressort que la distance Foyer Film a été respectée pour toutes les incidences de radiographie standard réalisée. L'utilisation de cache gonades a été faite toutes les fois qu'indiquées, bien que les tailles ne soient pas toujours adaptées, car plus larges dans $20 \%$ des cas. Dans 23,53\% (24) des cas, les images ont été reprises au moins une fois avec une présence parentale dans $58,33 \%$ de ces cas alors que les parents étaient hors de la salle d'examen dans $41,67 \%$. Les motifs de ces reprises de clichés étaient liés à l'utilisation de mauvais paramètres techniques dans 25 $\%$, un mauvais centrage dans $20,8 \%$ et une mauvaise coopération des enfants dans 13 cas soit $54,2 \%$.

\section{Les Valeurs Dosimetriques}

La Dose à l'entrée (De)

Le tableau III récapitule les valeurs de la Dose à l'entrée pour différents examens de Rx standard en fonction de la tranche d'âge et de la conformité ou non aux NRD français. 
Tableau III: Répartition des valeurs de De, en fonction de la tranche d’âge et la conformité ou non aux NRD français

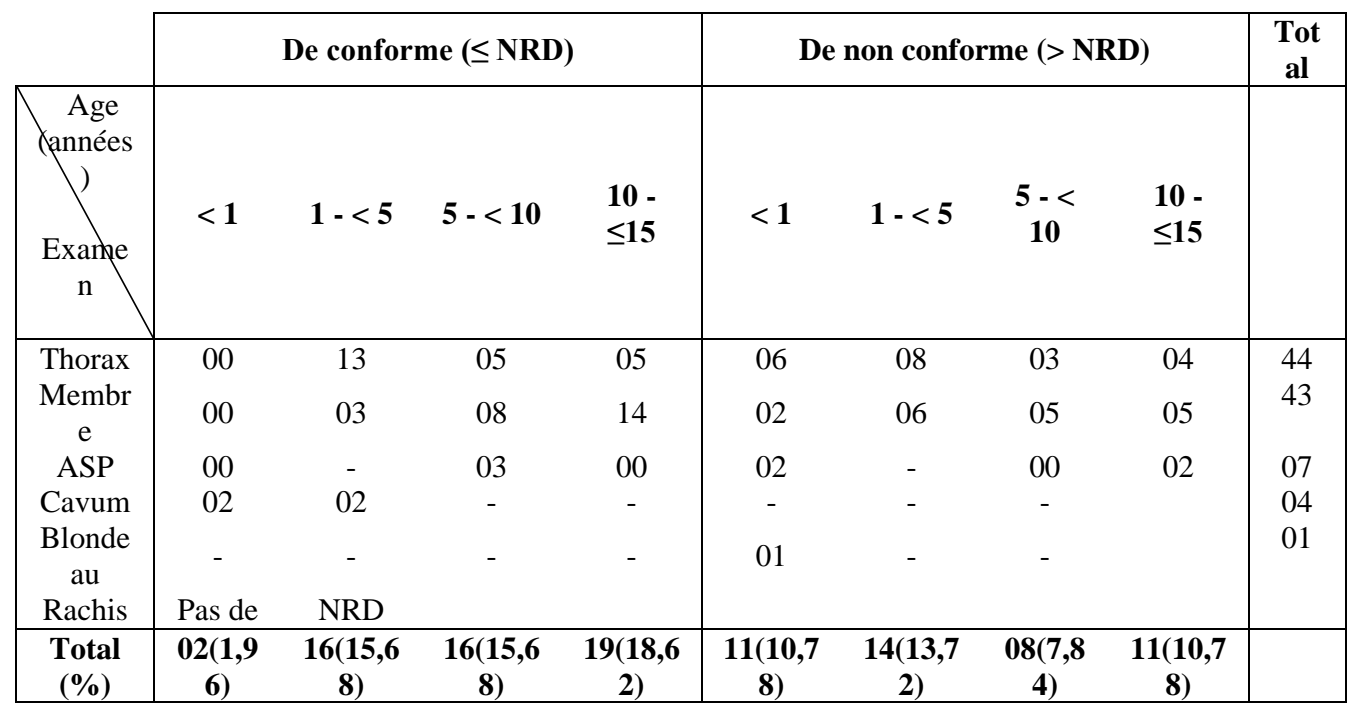

L'analyse des valeurs du PDS pour les différents examens de Rx standard en fonction de la tranche d'âge et de la conformité ou non aux NRD français est présentée sur le tableau IV.

Tableau IV: Répartition des valeurs du PDS, en fonction de la tranche d’âge et la conformité ou non aux NRD français

\begin{tabular}{|c|c|c|c|c|c|c|c|c|}
\hline & \multicolumn{4}{|c|}{ PDS conforme ( $\leq$ NRD) } & \multicolumn{4}{|c|}{ PDS non conforme (> NRD) } \\
\hline $\begin{array}{l}\text { Age } \\
\text { (années) }\end{array}$ & $<1$ & $1-<5$ & $5-<$ & $10-\leq 15$ & $<1$ & $1-<5$ & $5-<$ & $10-\leq 15$ \\
\hline Thorax & 00 & 04 & 05 & 02 & 06 & 17 & 03 & 07 \\
\hline Membre & Pas de & NRD & & & & & & \\
\hline ASP & 00 & 00 & 00 & & 02 & 03 & 02 & \\
\hline Cavum & Pas de & NRD & & & & & & \\
\hline Rachis & Pas de & NRD & & & & & & \\
\hline Blondeau & Pas de & NRD & & & & & & \\
\hline $\begin{array}{c}\text { Total } \\
(\%)\end{array}$ & 00(00) & $04(3,92)$ & $05(4,9)$ & $02(18,62)$ & $08(7,84)$ & 20(19,6) & $05(4,9)$ & 07(6,86) \\
\hline
\end{tabular}

\section{Discussion}

Notre étude avait porté sur l'analyse prospective observationnelle de 102 examens de radiographie réalisés sur une période de 6 mois dans le service de radiologie du CHU- Kara. Notre méthodologie présente certainement des insuffisances. En effet l'idéal aurait été d'effectuer des mesures directes sur fantômes dans une étude prospective à l'aide d'une 
chambre d'ionisation ou autre équipement de dosimétrie opérationnelle. De plus pour pouvoir faire des comparaisons avec les références existantes dans la littérature, nous ne nous sommes limités uniquement qu'à certaines régions anatomiques. Nous avons également exclu de notre étude les examens spéciaux de radiographie puisqu' il n'existe pas de niveaux de référence diagnostique (NRD) relatifs à ces examens chez l'enfant en France et en Belgique qui sont les pays dont nous avons utilisé les NRD. Toutefois, ces insuffisances ne sauraient enlever à ce travail sa valeur.

\section{Données épidémiologiques}

Le recours aux techniques d'imagerie, notamment à des techniques faisant appel à des rayonnements ionisants, se développe en pédiatrie, tant pour le diagnostic que pour le traitement des maladies de l'enfance (Organisation mondiale de la santé, 1987).

6,6 \% d'examens de radiographie standard étaient pratiqués chez les enfants dans le service de radiologie du CHU Kara, pendant notre période d'étude. Ce faible pourcentage pourrait s'expliquer par le fait que notre étude était unicentrique.

Les enfants de la tranche d'âge [5 - 10 ans [étaient les plus exposés aux rayons $\mathrm{X}$ en radiographie standard dans notre population d'étude, par opposition aux résultats d'Etard C (Etard C, 2014) où ceux de 10 à 15 ans et les bébés de la naissance à 1 an sont les populations les plus exposées de manière générale.

En radiopédiatrie, la radiographie du thorax constitue l'examen le plus fréquemment pratiqué sur les enfants du monde entier, représentant pas moins de $50 \%$ de l'imagerie diagnostique dans certaines régions (Organisation mondiale de la santé, 1987). C’est bien ce que notre travail retrouve puisque cet examen était le plus pratiqué. Selon une étude menée aux CHU de Lomé par Amouzou KB (2002), il représente 42,60\% de tous les examens, pourcentage presque égal au nôtre, 43 \% des examens de radiographie standard réalisés au CHU Kara pendant notre étude.

\section{Analyse de la justification}

Quatre-vingt (80) \% des examens de radiographie standard étaient bien justifiés selon le Guide de Bon Usage (GBU) des examens d'imagerie médicale. Une meilleure concertation entre les différents acteurs, comme indiquée par l’OMS (Organisation mondiale de la santé, 1987) contribuerait à augmenter ce pourcentage. En effet, les fournisseurs de soins de santé et qui demandent ces examens ont une responsabilité partagée en ce qui concerne le rapport bénéfice-risque. Une communication efficace et équilibrée est nécessaire et ses principes fondamentaux sont les mêmes pour tous les patients, mais une attention particulière est nécessaire dans le milieu 
pédiatrique (Broder J S et Frush D P, 2014) et la première préoccupation devrait être celle de ne pas réaliser un examen irradiant. Ceci est capital puisque l'allongement de l'espérance de vie chez les enfants permet plus de temps pour les effets nocifs des rayonnements de se manifester, et les organes et les tissus en développement sont plus sensibles aux effets des rayonnements (Khong PL et al. , 2013).

\section{Analyse de la procédure de réalisation des examens}

Une bonne technique radiographique chez l'enfant implique une adaptation de la procédure technique, de l'appareillage, du choix des paramètres et des accessoires éventuels.

La distance Foyer Film a été respectée pour toutes les incidences de radiographie standard réalisée. Il s'agit d'un paramètre géométrique qui influence la dose reçue par le patient et plus est grande, plus cette dose d'irradiation est diminuée.

L'utilisation de cache gonades a été faite toutes les fois qu'indiquées, bien que les tailles ne soient pas toujours adaptées, car plus larges dans $20 \%$ des cas, ce qui pourrait donc annuler ou diminuer l'efficacité de ce dispositif de radioprotection.

$\mathrm{La}$ reprise des examens radiographique est un important facteur évitable ou du moins réductible de surexposition des patients. 23,53\% des enfants avaient été exposés au moins deux fois. N’dakena G K et al. (2006) avaient trouvé $16,67 \%$, un pourcentage bien inférieur au nôtre mais leur travail n'a porté que sur la radiographie thoracique. En radiopédiatrie, le confort n'est pas un "luxe": l'inconfort physique ou psychologique est en effet source d'agitation et donc d'artéfacts. Cela conduit souvent à un maintien de l'enfant par l'un des parents ou par une tierce personne, auquel cas, il faut prévoir des protections pour les adultes présents dans la salle au cours de l'examen. En effet, la présence des parents est le plus souvent bénéfique permettant de rassurer l'enfant, aider à la contention ou encore donner un biberon. Toutes ces mesures sont indispensables pour que l'examen soit réalisé en toute sécurité (éviter les chutes de table) et avec la moindre irradiation (éviter de recommencer les clichés).

\section{Aspects dosimétriques}

Les niveaux de référence diagnostiques en radiologie, définis pour des examens courants, ne doivent pas être dépassés, sauf circonstances médicales particulières pour les procédures courantes, dès lors que les bonnes pratiques en matière de diagnostic et de performance technique sont appliquées (Journal officiel de la République Française, 2012).

En comparant nos résultats aux NRD françaises par tranche d'âge, $47,73 \%$ des radiographie du thorax, 41,86 \% des radiographie des membres 
et 57,14\% des radiographie de avaient des valeurs de De supérieures aux NRD françaises définies en fonction des tranches d’âge.

En ce qui concerne le PDS, 75\% des radiographies du thorax et 100\% des radiographies de l'ASP avaient des valeurs de DLP supérieures aux NRD belges définies en fonction des tranches d’âge. Ces valeurs dosimétriques largement au-dessus de normes européennes ont déjà été constatées dans des études récentes en Tomodensitométrie que ce soit chez les adultes (Tchaou M, 2015) ou chez les enfants (Tchaou M, 2016).

\section{Conclusion}

Cette étude révèle que du point de vue de la justification des examens radiologiques, la conformité des indications de ces examens avec le guide de bon usage des examens d'imagerie médicale n’est pas toujours effective mais satisfaisante en ce qui concerne la pratique radiopédiatrique au CHU Kara. L’étude des constantes dosimétriques montre que la dose délivrée aux enfants en radiographie standard excède dans une grande proportion les NRD français et belges surtout pour les radiographies du thorax et de l'ASP. L’analyse des différentes procédures montre qu'une réduction des reprises d'examens s'avère nécessaire.

La mise place d'un cadre réglementaire avec obligation de désignation et de formation de personnes compétente en radioprotection (PCR) permettrait de d'améliorer radioprotection des patients surtout les enfants mais aussi du personnel, ceci pour la mise en place de contrôle dosimétriques réguliers et des normes dosimétriques.

\section{References:}

AMOUZOU B K. (2002). Pratique de la radiologie conventionnelle aux CHU de Lomé, Mémoire, EAM-UB, Lomé, 2002.

BRODER J S, FRUSH D P. (2014). Content and style of radiation risk communication for pediatric patients. J Am Coll Radiol.; 11(3):238-42.

JOURNAL OFFICIEL DE LA REPUBLIQUE FRANÇAISE. (2012). Arrêté du 24 octobre 2011 relatif aux niveaux de référence diagnostiques en radiologie et en médecine nucléaire. JO du 14 Janvier 2012. p. 715.

KHONG PL, RINGERTZ H, DONOGHUE V, FRUSH D, et al. (2013). Radiological protection in paediatric diagnostic and interventional radiology. Ann ICRP; 42 (2):1-63.

ONGOLO-ZOGO P, MPEKE MOKUBANGELE C, MOIFO B. et al. (2012). Évaluation de la dose patient en scanographie pédiatrique dans deux hôpitaux universitaires à Yaoundé Cameroun. Radioprotection; 47(4) : 53342.

ORGANISATION MONDIALE DE LA SANTE (OMS). (1987). série de rapports techniques, $\mathrm{N}^{\circ} 757$ (utilisation rationnelle de l'imagerie diagnostique 
en pédiatrie : rapport d'un groupe d'étude de l'OMS), Genève, ISSN 0373$3998 ; 112 p$.

TCHAOU M, GNAKADJA G.N, N'TIMON B, L. et al. (2016) Revue des doses d'exposition et des méthodes d'optimisation en tomodensitométrie (TDM) de l'enfant au Togo. European Scientific Journal; 12 (6) : 73- 83. doi: 10.19044/esj.2016.v12n6p73

URL:http://dx.doi.org/10.19044/esj.2016.v12n6p73

TCHAOU M, SONHAYE L, AGODA - KOUSSEMA L K et al. (2015). Dosimétrie et radioprotection des patients lors des examens tomodensitométriques au CHU Sylvanus OLYMPIO (CHU-SO) de Lomé (Togo) . J. Rech. Sci. Univ. Lomé (Togo), Série D ; 17(1) : 387-95.

VROUSOS C, BERNARD AUBERT, DIETRICH AVERBECK et al. (2004). Priorités en radioprotection. Propositions pour une meilleure protection des personnes contre les dangers des rayonnements ionisants. Mars 2004. Accessible à l'URL http://www.ladocumentationfrancaise.fr/var/storage/rapportspublics/044000102.pdf. [Consulté le 10/12/2015] 